Highlights from the First Bi-monthly Virtual Meeting of Iranian Society of Cardiac Surgeons During COVID-19 Outbreak

\author{
Saeid Hosseini ${ }^{1}$, Hamidreza Taghipour ${ }^{2}$, Mohammad Reza Mirzaaghayan ${ }^{3}$ and Alireza Alizadeh \\ Ghavidel (iD ${ }^{1, *}$ \\ ${ }^{1}$ Heart Valve Disease Research Center, Rajaie Cardiovascular Medical and Research Center, Iran University of Medical Science, Tehran, Iran \\ ${ }^{2}$ Baghiyatollah General Hospital, Baghiyatollah University of Medical Science, Tehran, Iran \\ ${ }^{3}$ Tehran Children Hospital, Tehran University of Medical Science, Tehran, Iran \\ "Corresponding author: Heart Valve Disease Research Center, Rajaie Cardiovascular Medical and Research Center, Iran University of Medical Science, Vali-Asr St, Next to Mellat \\ Park, Tehran, Iran. Tel: +98-9121590518, Email: aaghavidel@gmail.com
}

Received 2020 June 21; Accepted 2020 June 24.

\begin{abstract} this article.

\section{Purpose of Meeting}

The Bi-monthly Meeting of the Iranian Society of Cardiac Surgeon (ISCS) has been held regularly since 2005. In this meeting, the Iranian cardiac surgeons present their challenging or problematic cases in order to find the best strategy for surgical management. Also some surgeons prefer to present their interesting or rare surgical cases or even their novel techniques in cardiac surgery. The first ISCS bi-monthly virtual meeting was held in May 2020 during the COVID-19 outbreak. We have briefly reported the content of this virtual meeting in this article.
\end{abstract}

The Bi-monthly Meeting of the Iranian Society of Cardiac Surgeon (ISCS) has been held regularly since 2005. The first ISCS bi-monthly virtual meeting was held in May 2020 during the COVID-19 outbreak. We have briefly reported the content of this virtual meeting in

Keywords: Endocarditis, CABG, MAC, Perceval S, Covid-19, Meeting Report, MV Repair, Valve Thrombosis, Tricupid Valve

\section{Summary of Presented Findings}

\subsection{CABG in a Suspicious COVID-19 Patient}

A 72-year-old gentleman presented with severe CAD and left main lesion, mild LV dysfunction (LVEF: 40\%) and good coronary artery anatomy, who was a candidate for urgent CABG due to ongoing ischemia. He had an unprotected contact with a COVID-19 positive patient without recent history of fever, chough, and other COVID-19 related symptoms. His chest X-Ray was unremarkable. White blood cell count was $8.3 \times 10^{9} / \mathrm{L}$ with lymph count $2100 / \mathrm{mm}$. Other lab tests including CRP were in normal ranges. Chest CT scan showed a nonspecific lung infiltration. The main question was about further evaluation of coronavirus involvement and surgical strategy. A couple of comments were given and some questions were asked and finally it was concluded that based on the latest evidences and ISCS protocol for COVID-19 patients, he should undergo urgent CABG without unnecessary delay (1). Based on each center's strategy, consultation with pulmonologist, infectious disease specialist, and COVID-19 PCR test sampling can be performed within few hours before the surgery. It is recommended that the patient's operations should be carried out in a COVID-19 dedicated operating theater with proper personal protecting equipment (PPE). It has also been emphasized that the patient and his/her family should be thoroughly informed about COVID-19 disease and underlying disease prognosis (1). After this question/answer section the patient's surgeon, presented his surgical plan and patient's short-term outcome that was uneventful.

\subsection{Prosthetic Valve Thrombosis in a Patient with COVID-19}

A 42-year-old lady with a mechanical valve in aortic position since 20 years ago and recent history of COVID-19 infection who was spending her home quarantine, had experienced progressive shortness of breath in the last 48 hours. She referred to a general hospital with pulmonary edema and had undergone transthoracic echocardiography and fluoroscopy that revealed valve thrombosis and severe restriction of leaflets motion with a very high peak 
transvalvular gradient $(110 \mathrm{mmHg})$. A mild anemia, borderline lymphocyte count $\left(1600 / \mathrm{mL}^{3}\right)$ and a positive quantitative CRP was found in lab tests. The interesting point was denying the COVID-19 infection by the patient and her family due to fear that the medical staff or surgical team would ignore the admission or surgery of the patient. She underwent emergent redo mechanical valve thrombectomy considering all protection principles and PPE without further preoperative evaluation. Her postoperative course was uneventful and weaning from mechanical ventilation was not challenging.

The surgical approach was mentioned and it was emphasized that during this pandemic; all patients who need emergent operations should be considered suspicious for SARS-Cov-2 virus infection and operated in a dedicated operating room with trained surgical team (1). COVID-19 PCR sampling and nonspecific lab tests including lymphocyte count and cell reactive protein (CRP) should be done preoperatively. These evaluations may affect patient management postoperatively. The patients should be transferred to the COVID dedicated wards if the PCR test results are positive for COVID-19.

\subsection{Mitral Valve Repair in a Young Lady with Severe MR and Severe MAC}

A 29-year-old young lady, known case of Marfan syndrome, candidate for mitral valve surgery due to her severe symptomatic mitral regurgitation was presented. She had severe mitral annular calcification (MAC) in preoperative echocardiography and heavy calcium bulk shadow in her chest $\mathrm{x}$-ray (Figure 1A). She had mild LV dysfunction and moderate pulmonary hypertension. The leaflets were pliable and the commissures were intact but multiscalop prolapse of mitral valve was evident as Barlow syndrome. Surgical plan A was mitral valve repair (MVr), however, surgical management of the severe MAC was definitely challenging. Surgical options including edge to edge repair (Alfieri technique), P2 prolapse repair and ring annuloplasty beyond the calcification, partial calcification de-bulking and mitral valve replacement (MVR) with multiple pledge ted sutures, complete removal of calcifications and creating a new annulus with pericardial patch and MVR were the recommended approaches. The surgeons' preferred option was detachment of posterior leaflet from the annulus, complete removal of the annular calcification, triangular resection of most prolaptic part in P2 segment, re-suturing of the detached posterior mitral leaflet to the corresponding annulus and ring annuloplasty by a semirigid incomplete ring (Figure $1 \mathrm{~B}$ and $\mathrm{C}$ ). The final result of surgery that was confirmed by post-procedure intraoperative TEE was trivial MR, no mitral stenosis and systolic anterior motion (SAM). The major concerns of most partic- ipants were the late outcome and recurrence of MR and also left atrio-ventricular groove injury, however favorable early and long term results have been reported and the rate of reported groove ruptures were not too high (2-4). It seems that for young patients with Myxomatous MR, complete removal of mitral annular calcification that needs mitral valve repair may be a good surgical option (5).

2.4. Sutureless Valve Implantation in an Old Patient in Combination with the Ascending Aorta Replacement, MVR and CABG

Favorable reports have been reported with sutureless Perceval (LivaNova, London, United Kingdom) bioprosthesis in recent years (6). Minimally invasive approaches could be a good alternative for aortic valve replacement (AVR) in high risk patients especially when transcatheter aortic valve implantation (TAVI) is not feasible. An 82-yearold man presented with severe symptomatic aortic stenosis (AS), coronary artery disease and severe MR. Median sternotomy and central aorto-right atrial appendage cannulation was used as surgical approach. Mitral valve replacement by a tissue valve and a three-graft CABG was performed. Supracoronary ascending aorta replacement using a tube Dacron graft was carried out and lastly a medium size Perceval bioprosthesis was implanted in aortic position. Intraoperative TEE confirmed proper position of the tissue valve and showed a $6 \mathrm{mmHg}$ transaortic gradient. The patients' postoperative course was uneventful and was discharged with normal sinus rhythm. Surgical challenges in this type of surgeries were discussed in this webinar and mentioned that the proper sizing of the valve is the key point for achieving optimal results. Oversizing may result in a significant malcoaptation of the bioprosthesis leaflet and significant residual aortic insufficiency that adversely affects the patients' early and late outcomes. The ratio of sinotubular junction to the annulus sizes is the other parameter for good surgical result and should be less than 1.3 that was 1.22 in the presented case. In high risk patients who need complex and multiple procedures, the use of sutureless valve may simplify the procedure and decrease the operation time.

The advantages and disadvantages of minimal approaches and the comparison of different techniques in incision (ministernotomy vs right anterior thoracotomy) and cannulation method (central vs peripheral) was also briefly discussed and it was concluded that case by case individualized approach should be considered based on surgeon's personal experience and patients' characteristics. Finally, the responsible surgeon briefly reported an off label implantation of Perceval in a dilated ascending aorta along with ascending aorta replacement. 

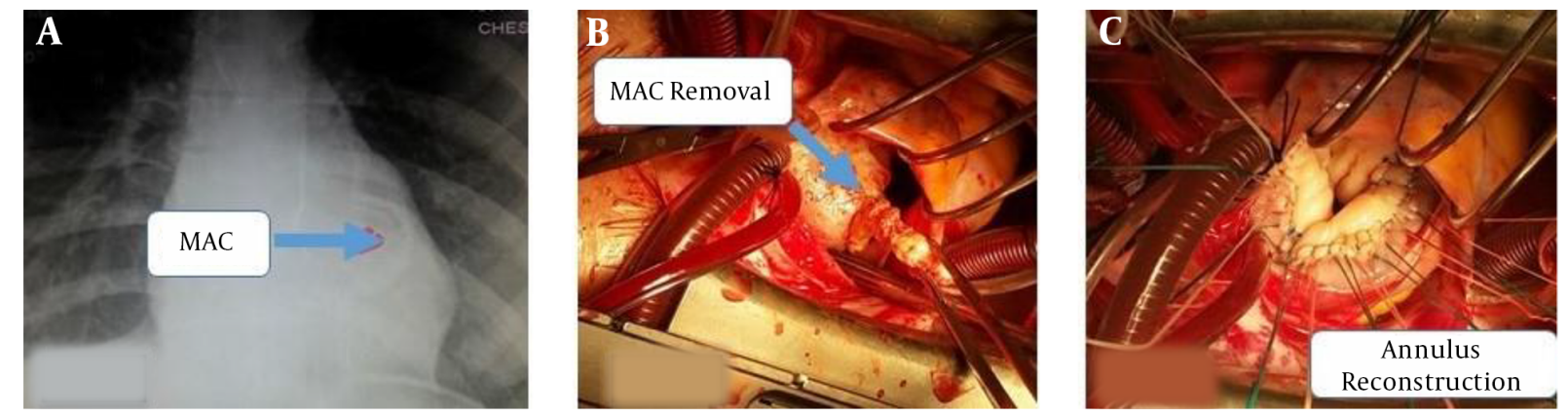

Figure 1. Mitral annulus calcification radiologic appearance and surgical resection and reconstruction. A, Sever mitral valve calcification in chest X-ray; B, mitral annulus calcification En-bloc resection from posterior leaflet; C, mitral annulus primary repair after removal of calcification.

\subsection{Tricuspid Valve Repair Using Autologous Pericardium in Na-} tive Valve Endocarditis

Durability and technical complexity are the two main concerns about tricuspid valve repair in endocarditis. On the other hand replacing the infected valve may lead to prosthetic valve endocarditis during short and mid-term follow-up that could be very challenging especially in patients that infection invaded the tricuspid valve annulus. Additionally, implanting a mechanical valve in tricuspid position composes the risk of prosthetic valve thrombosis that usually poorly tolerated or mandates a higher dose of oral anticoagulative and prothrombin time (PT) level that may adversely affect the patient's quality of life. Risk of bleeding and related complication is the other problematic issue in right-heart mechanical valves. Tissue valves in tricuspid position have the risk of early degeneration in young patients and re-intervention. Both types of prosthetic valves are also at risk of recurrent infection and secondary paravalvular leakage that adversely affect the patient's outcome. Overall, the timing of tricuspid valve surgery in patients with severe TR plays an important role in patient's late outcome (7), although a good surgical repair of infected tricuspid valve had been reported earlier by Dr. Hosseini et al. who presented the patient history in this webinar (8).

A case of native valve endocarditis presented in this meeting. She was a 23-year-old lady with a history of total parenteral nutrition (TPN) therapy with central venous catheter due to her GI tract fistula who admitted with fever and exertional dyspnea with the diagnosis of tricuspid valve endocarditis. She was scheduled for tricuspid valve surgery after a period of antibiotic therapy.

Radical debridement of infected valve tissue was done and new leaflets were fashioned using non-treated autologous pericardium (approximately 1.5 times larger than the corresponding leaflet) and 5 - 0 Prolene (PROLENE Polypropylene Suture, J \& J Medical Devices sutures). Then six neochorda were created by Gore-Tex CV 4 sutures (GORE-TEX ${ }^{\circledR}$ Suture-Gore) and ring annuloplasty was performed with a $32 \mathrm{~mm}$ MC3 rigid ring (Edward Lifesciences). Figure 2 demonstrates the surgical views of the different steps of valve repair. Intraoperative TEE and postoperative TTE revealed acceptable surgical results with mild tricuspid regurgitation and no stenosis. She was discharged with good status after three weeks of intravenous antibiotics and her early and midterm follow-up was uneventful.

\subsection{Catheter Related Atrial Thrombosis in a Young Child}

Catheter-related right atrial thrombosis (CRAT) is a well-known entity that may lead to lethal complication including pulmonary emboli and endocarditis that potentially has a high mortality rate around twenty percent. Non-surgical approaches including intravenous anticoagulation and fibrinolytic are usually effective in 30\% - 50\% of patients and surgical removal of the thrombotic or infected catheters is necessary for about $40 \%-50 \%$ of cases (9).

An 18-month-girl with acute lymphoblastic leukemia (ALL) was also presented in this webinar. She had a central venous port for chemotherapy since five months ago and presented with fever and malaise. She had a positive blood culture for pseudomonas aeruginosa and a $20 \mathrm{~mm}$ mass over the tip of port in junction of superior vena cava and right atrium in transthoracic echocardiography (Figure 3). The catheter was removed and a broad spectrum of antibiotics and full doses of unfractionated heparin were continued for one week, nevertheless the patient had recurrent fever. She was scheduled for surgical removal of the infected mass because the right atrial mass was still large and the medical management failed to treat the patient. Preoperative evaluations revealed a positive COVID19 PCR test, therefore the cardiac surgery was postponed. After a couple of days, she was re-scheduled for surgical removal of the infected mass but her parents refused the 

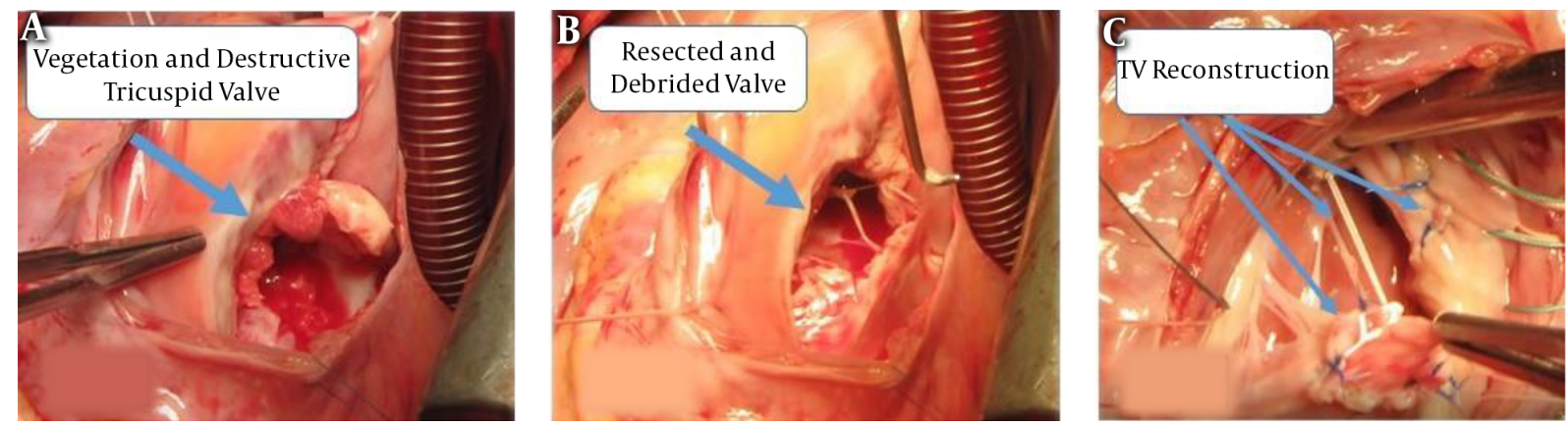

Figure 2. Surgical steps for Tricuspid valve reconstruction. A, Surgical view of the vegetation and destructive tricuspid valve; B, remnant of tricuspid valve after radical debridement; $C$, tricuspid valve reconstruction by pericardial patches and neochorda.

surgery. Therefore, conservative management was continued and lastly fever disappeared after one weeks. Control TTE revealed that the size of the mass has dramatically decreased. She was discharged without fever in a good status. The case presenter concluded that we should consider COVID-19 infection even in patients with underlying diseases that justify the patient fever and illness during pandemic. Furthermore urgent surgeries could be suspended during the acute phase of COVID-19 infection when possible.

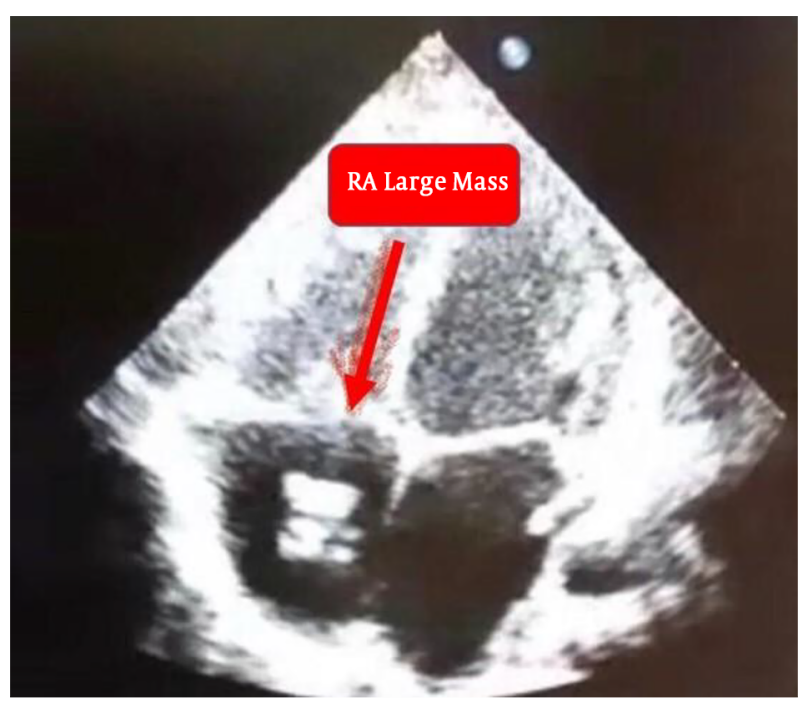

Figure 3. A large $20 \mathrm{~mm}$ right atrial mass in transthoracic echocardiography

\section{Acknowledgments}

We would like to thanks all participants of this webinar for their questions and comments during panel discussions.

\section{Footnotes}

Authors' Contribution: Saeid Hosseini did performing the surgeries of three presented cases and helping in manuscript drafting. Hamidreza Taghipour did performing the surgeries and presenting two cases in the webinar. Mohammad Reza Mirzaaghayan did management and presenting one case in the webinar, and reviewing the paper draft. Alireza Alizadeh Ghavidel did project concept, manuscript drafting, scientific editing, and also submitting the article.

Conflict of Interests: The authors declare no conflict of interest in these presentations.

Funding/Support: We declare that there was no funding support for the patients surgeries or manuscript preparation.

\section{References}

1. Asdaghpour E, Baghaei R, Jalilifar N, Radmehr H, Shirzad M, Mirzaaghayan MR, et al. Iranian Society of Cardiac Surgeons Position Statement for the treatment of patients in need of cardiac surgery in the COVID-19 pandemic period (version i). Multidiscip Cardiovasc Ann. 2020;11(1). doi: 10.5812/mca.104296.

2. Chan V, Ruel M, Hynes M, Chaudry S, Mesana TG. Impact of mitral annular calcification on early and late outcomes following mitral valve repair of myxomatous degeneration. Interact Cardiovasc Thorac Surg. 2013;17(1):120-5. doi: 10.1093/icvts/ivt163. [PubMed: 23587525]. [PubMed Central: PMC3686409].

3. Tomsic A, Hiemstra YL, van Brakel TJ, Versteegh MIM, Marsan NA, Klautz RJM, et al. Outcomes of valve repair for degenerative disease in patients with mitral annular calcification. Ann Thorac Surg. 2019;107(4):1195-201. doi: 10.1016/j.athoracsur.2018.08.017. [PubMed: 30296421].

4. Carino D, Agostinelli A, El Qarra S, Gripshi F, Nicolini F. Mitral annulus calcification: Current management and future challenges. Asian Cardiovasc Thorac Ann. 2019;27(7):565-72. doi: 10.1177/0218492319867237. [PubMed: 31342756].

5. Hosseini S, Samiei N, Mestres CA. The mitral annular stone: A surgical challenge. Eur J Cardiothorac Surg. 2015;48(5):805. doi: 10.1093/ejcts/ezv030. [PubMed: 25681420]. 
6. Alizadeh Ghavidel A, Sadeghpour A, Totonchi Z, Austin N, Aldairy A. Mid-term results of first experience in sutureless aortic valve replacement in Iran. Multidiscip Cardiovasc Ann. 2019;In Press(In Press). doi: 10.5812/mca.68611.

7. Sadeghpour A, Alizadehasl A, Azizi Z, Alizadeh Ghavidel A. Tricuspid regurgitation dilemma: A comparison study between surgical versus medical management of patients with tricuspid regurgitation. Multidiscip Cardio Annal. 2016;7(1). e9469. doi:10.17795/mcardia.9469.
8. Hosseini S, Rezaei Y, Mazaheri T, Almasi N, Babaei T, Mestres CA. Tricuspid valve repair for infective endocarditis with periannular involvement: Complete valve reconstruction. J Heart Valve Dis. 2016;25(6):730-8. [PubMed: 28290173].

9. Tran MH, Wilcox T, Tran PN. Catheter-related right atrial thrombosis. J Vasc Access. 2020;21(3):300-7. doi: 10.1177/1129729819873851. [PubMed: 31552793] 